\title{
The impact of filgotinib on patient-reported outcomes and health-related quality of life for patients with active rheumatoid arthritis: a post hoc analysis of Phase 3 studies
}

Clifton O. Bingham III ${ }^{1^{*}}$ (D, David Walker ${ }^{2}$, Peter Nash ${ }^{3}$, Susan J. Lee ${ }^{4}$, Lei Ye ${ }^{4}$, Hao Hu${ }^{4}$, Javaria Mona Khalid ${ }^{5}$ and Bernard Combe ${ }^{6}$

\begin{abstract}
Background: The effects of filgotinib on patient-reported outcomes (PROs) from 3 trials in patients with active rheumatoid arthritis were investigated.

Methods: Methotrexate (MTX)-naïve patients received filgotinib 200 or 100 mg plus MTX (FIL200+MTX, FIL100+MTX), filgotinib 200 mg monotherapy (FIL200), or MTX monotherapy through 52 weeks (NCT02886728). Patients with inadequate response (IR) to MTX (MTX-IR) received FIL200+MTX, FIL100+MTX, adalimumab 40 mg +MTX (ADA+MTX), or placebo (PBO)+MTX (rerandomized to FIL200+MTX or FIL100+MTX at week 24) through 52 weeks (NCT02889796). Patients with IR to biologic disease-modifying antirheumatic drugs (bDMARD-IR) received FIL200 or FIL100 or PBO with background stable conventional synthetic (cs) DMARDs for up to 24 weeks (NCT02873936). PROs included Health Assessment Questionnaire-Disability Index (HAQ-DI), Medical Outcomes Study 36-Item Short Form Health Survey (SF-36) physical/mental component summary (PCS/MCS), Functional Assessment of Chronic Illness Therapy-Fatigue (FACIT-Fatigue), Work Productivity and Activity Impairment Questionnaire-Rheumatoid Arthritis (WPAl-RA), and Patient Global Assessment of Disease Activity (PtGA). Data are reported as least-squares mean changes from baseline with standard error to the timepoint representing each study's primary endpoint. All statistical comparisons are of filgotinib groups vs their respective control groups.

Results: At week 24, among MTX-naïve patients, change from baseline (standard deviation) in HAQ-DI was - 1.00 $(0.03 ; P<0.001)$ with FIL200+MTX, $0.94(0.04 ; P<0.01)$ with FIL100+MTX, and $-0.91(0.04 ; P<0.05)$ with FIL200 alone compared with -0.81 (0.03) with MTX alone. At week 12, among MTX-IR patients, change from baseline in HAQ-DI was $-0.69(0.04 ; P<0.001$ vs PBO+MTX, $P<0.05$ vs ADA) with FIL200+MTX, $-0.57(0.04 ; P<0.001$ vs placebo) with FIL100+MTX, and - 0.60 (0.04) with ADA vs - 0.40 (0.04) with PBO+MTX. At week 12, among bDMARD-IR patients, change from baseline in HAQ-DI was $-0.50(0.06 ; P<0.001)$ with FIL200+CsDMARD and -0.46 $(0.05 ; P<0.001)$ with FIL100+csDMARD vs -0.19 (0.06) with placebo+csDMARD. Changes in SF-36 PCS and MCS, FACIT-Fatigue, WPAI, and PtGA tended to favor filgotinib over PBO, MTX, and ADA. Greater proportions of patients
\end{abstract}

\footnotetext{
*Correspondence: cbingha2@jhmi.edu

${ }^{1}$ Division of Rheumatology, Johns Hopkins University, 5200 Eastern

Avenue, Mason F. Lord Bldg, Center Tower, Room 434A, Baltimore, MD

21224, USA

Full list of author information is available at the end of the article
}

(c) The Author(s) 2021. Open Access This article is licensed under a Creative Commons Attribution 4.0 International License, which permits use, sharing, adaptation, distribution and reproduction in any medium or format, as long as you give appropriate credit to the original author(s) and the source, provide a link to the Creative Commons licence, and indicate if changes were made. The images or other third party material in this article are included in the article's Creative Commons licence, unless indicated otherwise in a credit line to the material. If material is not included in the article's Creative Commons licence and your intended use is not permitted by statutory regulation or exceeds the permitted use, you will need to obtain permission directly from the copyright holder. To view a copy of this licence, visit http://creativecommons.org/licenses/by/4.0/. The Creative Commons Public Domain Dedication waiver (http://creativeco mmons.org/publicdomain/zero/1.0/) applies to the data made available in this article, unless otherwise stated in a credit line to the data. 
experienced clinically meaningful differences with either dosage of FIL in combination with csDMARDs (including MTX) and with FIL200 monotherapy vs comparators.

Conclusions: Filgotinib provided improvements in PROs across patient populations. These findings suggest filgotinib can be an effective treatment option for patients with insufficient response to MTX or bDMARDs and patients who are MTX-naïve.

Trial registration: ClinicalTrials.gov, FINCH 1, NCT02889796, first posted September 7, 2016; FINCH 2, NCT02873936, first posted August 22, 2016, retrospectively registered; FINCH 3, NCT02886728, first posted September 1, 2016, retrospectively registered.

Keywords: Filgotinib, Patient-reported outcomes, HAQ-DI, SF-36, FACIT-Fatigue, WPAI

\section{Introduction}

Rheumatoid arthritis (RA) is an autoimmune disease in which immunologically driven inflammation leads to deterioration and impairment of joint tissue [1]. Functional impairment and chronic pain resulting from inflammation and progressive joint damage are common among patients with RA $[1,2]$. This impairment and pain often lead to disability; loss of joint function; inability to participate in desired family, social, and leisure activities; and reduced productivity at work, all of which negatively impact health-related quality of life (HRQL) $[1,2]$. HRQL assessments have become an important part of many clinical RA studies to better evaluate the patient experience and the impact of treatment on patient well-being [3, 4].

Filgotinib is a once-daily, oral, Janus kinase 1 (JAK1) preferential inhibitor [5]. In three Phase 3 studies, filgotinib improved signs and symptoms of RA and physical function in patients with inadequate response to methotrexate (MTX-IR; FINCH 1, NCT02889796) or biologic disease-modifying antirheumatic drugs (bDMARD-IR; FINCH 2, NCT02873936), and in patients with early RA who had not been treated with MTX (MTX-naive; FINCH 3, NCT02886728) [1, 6, 7]. In these studies, higher proportions of patients achieved the primary endpoint of $20 \%$ improvement in American College of Rheumatology (ACR20) criteria relative to placebo. The present analysis evaluated the effect of filgotinib on measures of HRQL in patients with RA by analyzing patient-reported outcomes (PROs) captured during these clinical trials, including the Health Assessment Questionnaire-Disability Index (HAQ-DI), Medical Outcomes Study 36-Item Short Form Survey (SF-36), Functional Assessment of Chronic Illness Therapy-Fatigue (FACITFatigue), Work Productivity and Activity Impairment Questionnaire-Rheumatoid Arthritis (WPAI-RA), and Patient Global Assessment of Disease Activity (PtGA).

\section{Methods}

\section{Study design}

All 3 trials were randomized, double-blind, placebo- (MTX-IR, bDMARD-IR) or active-controlled (adalimumab for the MTX-IR trial and MTX for the MTX-naïve trial), multicenter, Phase 3 studies. The trials were conducted in accordance with the Declaration of Helsinki and International Council for Harmonisation Good Clinical Practice guidelines. The study protocols were approved by the international review board or ethics committee at each study site, and all patients provided written informed consent. This is a post hoc analysis of PROs.

Details of the studies have been reported previously $[1,6,7]$. Patients were aged $\geq 18$ years with $\geq 6$ swollen joints (from swollen joint count of 66 joints) and $\geq$ 6 tender joints (from tender joint count of 68 joints) at screening and baseline. MTX-naïve patients had limited or no prior treatment with MTX (no more than 3 doses of MTX $\leq 25 \mathrm{mg}$ each in the patient's lifetime for the treatment of RA), with the last dose at least 28 days prior to study day 1 . Patients in this trial were randomized 2:1:1:2 to filgotinib $200 \mathrm{mg}$ plus MTX, filgotinib $100 \mathrm{mg}$ plus MTX, filgotinib $200 \mathrm{mg}$ monotherapy, or MTX monotherapy administered for up to 52 weeks. The time point for the primary endpoint-the proportion of patients who achieved ACR20 response-was week 24. In the MTX-IR trial, patients receiving ongoing MTX treatment for $\geq 12$ weeks at a dose of 7.5 to $25 \mathrm{mg} /$ week were randomized 3:3:2:3 to receive once daily filgotinib $200 \mathrm{mg}$, filgotinib $100 \mathrm{mg}$, subcutaneous adalimumab $40 \mathrm{mg}$, or placebo for up to 52 weeks with a stable weekly background of MTX. At week 24, patients receiving placebo who did not discontinue treatment were rerandomized $1: 1$ to filgotinib 200 or $100 \mathrm{mg}$. The time point for the primary endpoint-the proportion of patients who achieved ACR20 response-was week 12 . Patients in the bDMARD-IR trial were randomized 1:1:1 to receive once-daily filgotinib $200 \mathrm{mg}$, filgotinib 100 $\mathrm{mg}$, or placebo for up to 24 weeks, all on background stable dosages of conventional synthetic (cs) DMARDs. The time point for the primary endpoint-the proportion of patients who achieved ACR20 response-was week 12 . 


\section{Patient-reported outcomes}

HAQ-DI, SF-36, FACIT-Fatigue, WPAI-RA, and PtGA were collected prospectively throughout the studies. HAQ-DI assesses 8 functional categories (dressing and grooming, arising, eating, walking, hygiene, reach, grip, and other activities), with scores ranging from 0 (no disability) to 3 (completely disabled) [8-10]. The SF-36 is a 36-item questionnaire grouped into 8 scales, which can be further summarized as Physical and Mental Component scores; scores range from 0 to 100, representing "least health" to "greatest health." [11] FACIT-Fatigue is a 13-item questionnaire concerning fatigue severity and impact over the previous week that is scored from 0 to 52, with higher scores indicating less fatigue [11]. WPAI is a quantitative assessment of the amount of absenteeism, presenteeism, and daily activity impairment attributable to general health or a specific health problem [12, 13]. The RA-specific version, WPAI-RA, is a self-administered 6-item questionnaire; activity impairment is a single item score evaluated among all patients, while work productivity impairment (employed patients only) is a weighted score of absenteeism and presenteeism. Negative values represent improvement [14]. PtGA uses a visual analog scale ranging from 0 to $100 \mathrm{~mm}$; higher scores indicate worse disease [1]. These questionnaires have been validated and used extensively in RA clinical trials [8-11, 14-18].

In all three Phase 3 studies, HAQ-DI and PtGA were assessed at baseline and at weeks 2, 4, 8, 12, 16, 20, and 24. In the MTX-IR and MTX-naive trials, assessments were also made at weeks $30,36,44$, and 52 . SF-36, FACIT-Fatigue, and WPAI-RA were collected at baseline and weeks 4,12 , and 24 , as well as weeks 36 and 52 for the MTX-IR and MTX-naïve trials. The minimally clinically important difference (MCID) was defined as achievement of $\mathrm{a} \geq 0.22$ point reduction from baseline for HAQDI, $a \geq 2.5$-point increase from baseline for SF-36 Mental Component Score (MCS) and Physical Component Score (PCS), and $\mathrm{a} \geq 4$-point increase from baseline for FACITFatigue $[11,19,20]$.

\section{Statistical analyses}

Mean changes from baseline in HAQ-DI, SF-36, FACITFatigue, WPAI, and PtGA for each filgotinib arm were compared with mean changes from baseline in placebo (MTX-IR and bDMARD-IR trials), adalimumab (MTXIR trial), or MTX (MTX-naïve trial) using the mixedeffects model for repeated measures (MMRM), including treatment group, visit, treatment group by visit interaction, stratification factors (including geographic region, presence of rheumatoid factor or anti-cyclic citrullinated peptide antibodies, and prior exposure to bDMARDs), and baseline value of the measure being tested as fixed effects, and patients as random effect. Missing change scores due to missing study visits or early withdrawal were not otherwise imputed using the MMRM approach.

Additional analyses included the proportion of patients with HAQ-DI $\leq 0.5$ and the proportion of patients achieving HAQ-DI, SF-36, and FACIT-Fatigue MCID. The proportion of patients achieving these thresholds with each filgotinib regimen and with placebo (MTXIR and bDMARD-IR trials), adalimumab (MTX-IR), or MTX (MTX-naïve) were compared using a logistic regression model including treatment group and stratification factors. A nonresponder imputation was used for patients with missing data. All analyses were exploratory without multiplicity adjustment, and exploratory $P$ values are reported.

\section{Results}

Baseline demographics and disease characteristics

Of the patients randomized and treated, 1025/1249 (82.1\%) MTX-naïve patients, 1517/1755 (86.4\%) MTXIR patients, and 381/448 (85.0\%) bDMARD-IR patients completed their studies. Patient demographics and baseline disease characteristics were comparable between the treatment groups in each study and are listed in Supplemental Tables 1, 2 and 3. Baseline PRO scores were similar between treatment groups in each study and indicated a substantial disease burden (Supplemental Tables 1, 2 and 3).

\section{Differences between treatment groups in HAQ-DI changes from baseline}

At week 4, improvements in HAQ-DI score from baseline were greater for MTX-naïve patients who received filgotinib $200 \mathrm{mg}$ plus MTX $(-0.64 \pm 0.03)$, filgotinib $100 \mathrm{mg}$ plus MTX $(-0.50 \pm 0.04)$, or filgotinib $200 \mathrm{mg}$ monotherapy $(-0.56 \pm 0.04)$ than with MTX alone $(-0.35$ $\pm 0.03, P<0.001$ for all filgotinib treatments vs MTX alone) (Table 1). Improvements were significantly greater for those receiving any dose or treatment regimen of filgotinib relative to MTX alone at week 24 , and improvements remained greater for filgotinib $200 \mathrm{mg}$ plus MTX and filgotinib $200 \mathrm{mg}$ monotherapy compared to MTX alone at week 52. Improvements for patients receiving filgotinib $100 \mathrm{mg}$ plus MTX and MTX alone were similar at week 52 .

Among MTX-IR patients, improvements in HAQ-DI were greater at weeks 4,12 , and 24 for patients receiving filgotinib $200 \mathrm{mg}$ plus MTX and $100 \mathrm{mg}$ plus MTX compared to placebo. Improvements in HAQ-DI at week 4 were $-0.44 \pm 0.04$ for patients receiving filgotinib 200 $\mathrm{mg}$ and $-0.35 \pm 0.04$ for patients receiving filgotinib 100 mg compared to $-0.25 \pm 0.04$ for placebo $(P<0.001$ for both) (Table 2). HAQ-DI improvements were similar for 
Table 1 Least-squares mean change from baseline for patient-reported outcomes in MTX-naïve patients; filgotinib dosing regimens compared to MTX

\begin{tabular}{|c|c|c|c|c|}
\hline PRO measures & FIL $200 \mathrm{mg}+$ MTX $n=416$ & FIL $100 \mathrm{mg}+$ MTX $n=207$ & FIL $200 \mathrm{mg} n=210$ & MTX $n=416$ \\
\hline \multicolumn{5}{|l|}{ HAQ-DI } \\
\hline Week 4 & $-0.64(0.03)^{* * * *}$ & $-0.50(0.04)^{* * * *}$ & $-0.56(0.04)^{* * *}$ & $-0.35(0.03)$ \\
\hline Week 12 & $-0.91(0.03)^{* * *}$ & $-0.82(0.04)^{* * * *}$ & $-0.80(0.04)^{* * *}$ & $-0.64(0.03)$ \\
\hline Week 24 & $-1.00(0.03)^{* * *}$ & $-0.94(0.04)^{* *}$ & $-0.91(0.04)^{*}$ & $-0.81(0.03)$ \\
\hline Week 52 & $-1.03(0.04)^{* * * *}$ & $-0.96(0.05)$ & $-0.98(0.05)^{*}$ & $-0.87(0.04)$ \\
\hline \multicolumn{5}{|l|}{ SF-36 PCS } \\
\hline Week 4 & $7.3(0.4)^{* * * *}$ & $5.8(0.5)^{* * *}$ & $6.4(0.5)^{* * * *}$ & $4.1(0.4)$ \\
\hline Week 12 & $11.6(0.4)^{* * * *}$ & $9.6(0.6)^{* * *}$ & $9.4(0.6)^{*}$ & $7.9(0.4)$ \\
\hline Week 24 & $12.8(0.5)^{* * *}$ & $11.5(0.6)^{*}$ & $10.8(0.6)$ & $9.9(0.5)$ \\
\hline Week 52 & $13.6(0.5)^{* * * *}$ & $12.0(0.7)$ & $12.2(0.7)$ & $10.8(0.5)$ \\
\hline \multicolumn{5}{|l|}{ SF-36 MCS } \\
\hline Week 4 & $4.6(0.5)^{* * *}$ & $3.4(0.6)^{*}$ & $4.3(0.6)^{* * *}$ & $1.9(0.5)$ \\
\hline Week 12 & $5.7(0.5)^{*}$ & $5.7(0.7)$ & $5.4(0.7)$ & $4.3(0.5)$ \\
\hline Week 24 & $5.9(0.5)$ & $6.3(0.7)$ & $5.5(0.7)$ & $5.7(0.5)$ \\
\hline Week 52 & $6.5(0.5)$ & $6.2(0.7)$ & $5.8(0.7)$ & $5.8(0.6)$ \\
\hline \multicolumn{5}{|l|}{ FACIT-Fatigue } \\
\hline Week 4 & $7.6(0.5)^{* * * *}$ & $6.9(0.6)^{* * *}$ & $7.1(0.6)^{* * * *}$ & $4.4(0.5)$ \\
\hline Week 12 & $10.3(0.5)^{* * * *}$ & $9.3(0.7)$ & $9.8(0.7)^{*}$ & $8.1(0.5)$ \\
\hline Week 24 & $11.3(0.6)$ & $11.3(0.7)$ & $10.3(0.7)$ & $10.0(0.6)$ \\
\hline Week 52 & $12.1(0.6)^{*}$ & $11.4(0.8)$ & $11.6(0.8)$ & $10.4(0.6)$ \\
\hline \multicolumn{5}{|l|}{ PtGA } \\
\hline Week 4 & $-28(1.2)^{* * * *}$ & $-21(1.6)^{* *}$ & $-23(1.6)^{* * *}$ & $-15(1.2)$ \\
\hline Week 12 & $-38(1.3)^{* * *}$ & $-31(1.7)^{* *}$ & $-32(1.7)^{* *}$ & $-26(1.3)$ \\
\hline Week 24 & $-44(1.3)^{* * *}$ & $-38(1.7)$ & $-38(1.7)$ & $-34(1.3)$ \\
\hline Week 52 & $-46(1.4)^{* * * *}$ & $-40(1.8)$ & $-43(1.8)^{* *}$ & $-37(1.4)$ \\
\hline \multicolumn{5}{|l|}{ WPAI-RA } \\
\hline \multicolumn{5}{|l|}{ Absenteeism } \\
\hline Week 4 & $-2.9(2.0)$ & $-0.3(2.8)$ & $-4.8(2.7)$ & $-0.2(2.1)$ \\
\hline Week 12 & $-6.2(1.9)$ & $-8.3(2.6)$ & $-2.9(2.4)$ & $-3.8(1.8)$ \\
\hline Week 24 & $-6.7(1.7)$ & $-10.2(2.3)$ & $-3.6(2.1)^{*}$ & $-9.0(1.7)$ \\
\hline Week 52 & $-7.0(1.8)$ & $-7.9(2.5)$ & $-4.2(2.4)$ & $-7.6(1.9)$ \\
\hline \multicolumn{5}{|c|}{ Presenteeism } \\
\hline Week 4 & $-19.4(2.0)^{* * *}$ & $-22.3(2.8)^{* * *}$ & $-17.3(2.6)^{* * *}$ & $-7.2(2.1)$ \\
\hline Week 12 & $-27.2(2.0)^{* *}$ & $-28.2(2.8)^{*}$ & $-25.4(2.6)$ & $-19.9(2.0)$ \\
\hline Week 24 & $-29.8(1.9)$ & $-32.2(2.7)$ & $-27.5(2.5)$ & $-26.8(2.0)$ \\
\hline Week 52 & $-33.6(1.9)$ & $-30.7(2.7)$ & $-32.2(2.5)$ & $-30.0(2.1)$ \\
\hline \multicolumn{5}{|c|}{ Work productivity loss } \\
\hline Week 4 & $-19.0(2.1)^{* * *}$ & $-21.8(3.0)^{* * *}$ & $-17.1(2.8)^{* * *}$ & $-6.6(2.2)$ \\
\hline Week 12 & $-27.6(2.2)^{* *}$ & $-28.1(3.1)^{*}$ & $-23.4(2.9)$ & $-19.5(2.2)$ \\
\hline Week 24 & $-30.3(2.1)$ & $-30.5(3.0)$ & $-24.6(2.8)$ & $-26.4(2.2)$ \\
\hline Week 52 & $-33.4(2.2)$ & $-31.2(3.1)$ & $-29.8(2.9)$ & $-30.3(2.4)$ \\
\hline \multicolumn{5}{|c|}{ Activity impairment } \\
\hline Week 4 & $-23.2(1.3)^{* * *}$ & $-17.8(1.7)^{*}$ & $-19.7(1.7)^{* *}$ & $-13.5(1.3)$ \\
\hline Week 12 & $-32.5(1.4)^{* * * *}$ & $-28.1(1.8)^{*}$ & $-30.5(1.8)^{k * * *}$ & $-23.6(1.4)$ \\
\hline Week 24 & $-36.4(1.4)^{*}$ & $-34.6(1.8)$ & $-32.7(1.8)$ & $-32.3(1.4)$ \\
\hline Week 52 & $-39.9(1.4)^{* * *}$ & $-36.4(1.9)$ & $-38.4(1.9)$ & $-34.5(1.5)$ \\
\hline
\end{tabular}

Data presented as LS mean (SE). The primary endpoint was assessed at Week 24. Comparison with methotrexate: ${ }^{* * *} P<0.001,{ }^{* *} P<0.01,{ }^{*} P<0.05$. All $P$ values are exploratory (not adjusted for multiplicity) and were assessed using the MMRM, including treatment group, visit, treatment group by visit interaction, stratification factors, and baseline value as fixed effects, and patients as random effect.

FACIT, Functional Assessment of Chronic Illness Therapy; FIL, filgotinib; HAQ-DI, Health Assessment Questionnaire-Disability Index; LS, least-squares; MCS, Mental Component Score; MTX, methotrexate; PCS, Physical Component Score; PRO, patient-reported outcomes; PtGA, Patient Global Assessment of Disease Activity; SE, standard error; SF-36, Medical Outcomes Study 36-Item Short Form; WPAI-RA, Work Productivity and Activity Impairment Questionnaire-Rheumatoid Arthritis 
Table 2 Least-squares mean change from baseline for patient-reported outcomes in MTX-IR patients; filgotinib dosing regimens compared to placebo and adalimumab

\begin{tabular}{|c|c|c|c|c|}
\hline PRO measures & FIL $200 \mathrm{mg}+$ MTX $n=475$ & FIL $100 \mathrm{mg}+\mathrm{MTX} n=480$ & $\mathrm{ADA}+\mathrm{MTX} n=325$ & $\mathrm{PBO} n=475$ \\
\hline \multicolumn{5}{|l|}{ HAQ-DI } \\
\hline Week 4 & $-0.44(0.04)^{* * *}$ & $-0.35(0.04)^{* * *}$ & $-0.40(0.04)$ & $-0.25(0.04)$ \\
\hline Week 12 & $-0.69(0.04)^{* * *+}$ & $-0.57(0.04)^{* * *}$ & $-0.60(0.04)$ & $-0.40(0.04)$ \\
\hline Week 24 & $-0.82(0.04)^{* * *}$ & $-0.74(0.04)^{* * *}$ & $-0.76(0.04)$ & $-0.55(0.04)$ \\
\hline Week 52 & $-0.90(0.04)^{\dagger}$ & $-0.82(0.04)$ & $-0.82(0.05)$ & - \\
\hline \multicolumn{5}{|l|}{ SF-36 PCS } \\
\hline Week 4 & $6.0(0.5)^{* * *+}$ & $5.1(0.5)^{* * *}$ & $5.1(0.6)$ & $3.4(0.6)$ \\
\hline Week 12 & $9.7(0.6)^{* * *+}$ & $9.1(0.6)^{* * * *}$ & $8.6(0.6)$ & $5.9(0.6)$ \\
\hline Week 24 & $10.7(0.6)^{* * *}$ & $10.6(0.6)^{* * *}$ & $10.3(0.6)$ & $7.6(0.6)$ \\
\hline Week 52 & $12.0(0.6)$ & $11.6(0.6)$ & $11.9(0.7)$ & - \\
\hline \multicolumn{5}{|l|}{ SF-36 MCS } \\
\hline Week 4 & $3.3(0.6)^{* * *}$ & $3.0(0.6)^{* *}$ & $3.2(0.7)$ & $1.5(0.6)$ \\
\hline Week 12 & $4.8(0.6)^{* *}$ & $5.0(0.6)^{* *}$ & $4.2(0.7)$ & $3.3(0.6)$ \\
\hline Week 24 & $5.4(0.6)$ & $5.3(0.6)$ & $4.7(0.7)$ & $4.4(0.7)$ \\
\hline Week 52 & $5.7(0.7)$ & $6.2(0.7)$ & $5.5(0.7)$ & - \\
\hline \multicolumn{5}{|l|}{ FACIT-Fatigue } \\
\hline Week 4 & $6.5(0.7)^{* * *}$ & $5.9(0.7)^{* * *}$ & $5.7(0.7)$ & $3.7(0.7)$ \\
\hline Week 12 & $9.4(0.7)^{* * * *}$ & $9.3(0.7)^{* * *}$ & $8.8(0.7)$ & $6.6(0.7)$ \\
\hline Week 24 & $10.7(0.7)^{* * *}$ & $10.8(0.7)^{* * *}$ & $10.0(0.8)$ & $8.0(0.7)$ \\
\hline Week 52 & $11.7(0.7)$ & $11.8(0.7)$ & $10.9(0.8)$ & - \\
\hline \multicolumn{5}{|l|}{ PtGA } \\
\hline Week 4 & $-23(1.5)^{* * *}$ & $-18(1.5)^{* * *}$ & $-21(1.6)$ & $-14(1.6)$ \\
\hline Week 12 & $-34(1.6)^{* * *+}$ & $-30(1.6)^{* * *}$ & $-29(1.8)$ & $-21(1.6)$ \\
\hline Week 24 & $-40(1.6)^{* * *}$ & $-37(1.6)^{* * *}$ & $-37(1.8)$ & $-29(1.7)$ \\
\hline Week 52 & $-43(1.7)$ & $-40(1.7)$ & $-41(1.9)$ & - \\
\hline \multicolumn{5}{|l|}{ WPAI-RA } \\
\hline \multicolumn{5}{|l|}{ Absenteeism } \\
\hline Week 4 & $0.6(2.1)$ & $-1.3(2.1)$ & $-0.9(2.4)$ & $-0.4(2.2)$ \\
\hline Week 12 & $-2.3(2.0)$ & $-2.7(2.0)$ & $-2.1(2.3)$ & $-0.2(2.2)$ \\
\hline Week 24 & $-3.0(2.0)^{* *}$ & $-3.5(2.0)^{* *}$ & $-2.3(2.3)$ & $2.2(2.2)$ \\
\hline Week 52 & $-1.5(1.8)$ & $-0.0(1.9)$ & $0.6(2.2)$ & - \\
\hline \multicolumn{5}{|l|}{ Presenteeism } \\
\hline Week 4 & $-13.1(2.6)^{* *}$ & $-9.0(2.5)$ & $-11.4(2.9)$ & $-6.1(2.7)$ \\
\hline Week 12 & $-20.9(2.6)^{* * *}$ & $-20.2(2.5)^{* *}$ & $-20.3(2.9)$ & $-13.0(2.8)$ \\
\hline Week 24 & $-25.2(2.6)^{* * *}$ & $-23.8(2.6)^{* *}$ & $-21.0(3.0)$ & $-16.3(2.9)$ \\
\hline Week 52 & $-28.6(2.5)$ & $-27.0(2.5)$ & $-25.3(2.9)$ & - \\
\hline \multicolumn{5}{|c|}{ Work productivity loss } \\
\hline Week 4 & $-11.7(2.8)^{* *}$ & $-7.9(2.7)$ & $-10.9(3.1)$ & $-5.3(2.9)$ \\
\hline Week 12 & $-19.3(2.8)^{* *}$ & $-19.2(2.7)^{* *}$ & $-18.5(3.2)$ & $-11.6(3.0)$ \\
\hline Week 24 & $-23.8(2.9)^{* * *}$ & $-22.9(2.8)^{* * *}$ & $-19.2(3.3)$ & $-13.4(3.1)$ \\
\hline Week 52 & $-26.8(2.8)$ & $-24.4(2.8)$ & $-22.7(3.2)$ & - \\
\hline \multicolumn{5}{|c|}{ Activity impairment } \\
\hline Week 4 & $-18.0(1.7)^{* * *}$ & $-16.1(1.7)^{* * *}$ & $-16.0(1.8)$ & $-10.6(1.7)$ \\
\hline Week 12 & $-27.3(1.7)^{* * * \dagger}$ & $-25.6(1.7)^{* * * *}$ & $-23.7(1.9)$ & $-17.9(1.8)$ \\
\hline Week 24 & $-31.5(1.7)^{* * *}$ & $-31.3(1.8)^{* * *}$ & $-29.2(1.9)$ & $-22.0(1.8)$ \\
\hline Week 52 & $-35.4(1.8)$ & $-35.1(1.8)$ & $-33.1(2.0)$ & - \\
\hline
\end{tabular}

Data presented as LS mean (SE). The primary endpoint was assessed at Week 12. Comparison with placebo: ${ }^{* * *} P<0.001,{ }^{* * *} P<0.01$. Comparison with adalimumab: ${ }^{\dagger} P$ $<0.05$. All $P$ values are exploratory (not adjusted for multiplicity) and assessed using the MMRM, including treatment group, visit, treatment group by visit interaction, stratification factors, baseline value as fixed effects, and patients as random effect. In FINCH 1, patients on PBO were rerandomized to FIL 200 or $100 \mathrm{mg}$ at week 24. $A D A$, adalimumab; FACIT, Functional Assessment of Chronic Illness Therapy; FIL, filgotinib; LS, least-squares; HAQ-DI, Health Assessment Questionnaire-Disability Index; 
Table 2 (continued)

MCS, Mental Component Score; MTX, methotrexate; PBO, placebo; PCS, Physical Component Score; PRO, patient-reported outcomes; PtGA, Patient Global Assessment of Disease Activity; SE, standard error; SF-36, Medical Outcomes Study 36-Item Short Form; WPAl-RA, Work Productivity and Activity Impairment Questionnaire-Rheumatoid Arthritis

patients receiving filgotinib $200 \mathrm{mg}$ plus MTX at weeks 4 and 24 relative to adalimumab plus MTX, while improvements from baseline were greater among patients who received filgotinib $200 \mathrm{mg}$ plus MTX relative to adalimumab plus MTX at weeks 12 and 52 (Table 2). For filgotinib $100 \mathrm{mg}$ plus MTX, improvements from baseline were similar throughout the study compared with patients receiving adalimumab plus MTX.

Patients who were bDMARD-IR and on a background of csDMARDswho received filgotinib $200(-0.35 \pm 0.05)$ and $100 \mathrm{mg}(-0.28 \pm 0.05)$ reported improvements relative to placebo $(-0.13 \pm 0.05, P<0.001$ and $P=0.006)$ at week 4 . Significant improvements were maintained through week 24(Table 3).

\section{Differences between treatment groups in proportions of patients achieving HAQ-DI MCID}

The proportion of patients who achieved MCID for HAQ-DI- $\mathrm{a} \geq 0.22$-point reduction from baselineincreased starting at week 4 in all patient populations. Among MTX-naïve patients at week 4, 72.4\% who received filgotinib $200 \mathrm{mg}$ plus MTX $(P<0.001)$, $61.0 \%$ who received filgotinib $100 \mathrm{mg}$ plus MTX $(P=$ $0.083)$, and $68.6 \%$ who received filgotinib $200 \mathrm{mg}(P<$ 0.001 ) achieved MCID compared to $53.9 \%$ of patients who received MTX alone. At week 24, the proportion achieving MCID was similar between patients receiving any dosing regimen of filgotinib and patients receiving MTX alone. Greater proportions of patients who received any dosage of filgotinib achieved MCID relative to those who received MTX monotherapy at week 52 (Fig. 1A).

At week $4,66.2 \%$ of MTX-IR patients who received filgotinib $200 \mathrm{mg}(P<0.001)$ and $58.0 \%$ of those who received filgotinib $100 \mathrm{mg}(P=0.011)$ achieved MCID compared to $49.9 \%$ of patients who received placebo (Fig. 1B). At weeks 12 and 24, the proportion of MTX-IR patients achieving MCID remained significantly greater for patients receiving either dose of filgotinib relative to placebo. A greater proportion of MTX-IR patients who received filgotinib $200 \mathrm{mg}$ achieved MCID at week 12 than those who received adalimumab $(78.9 \%$ vs $72.8 \%, P$ $=0.046$ ); the proportion remained numerically greater through week 52 (Fig. 1B). The percentage of MTX-IR patients achieving MCID for HAQ-DI was numerically greater for patients who received filgotinib $100 \mathrm{mg}$ relative to adalimumab throughout the study (Fig. 1B).
At all timepoints, a greater proportion of bDMARDIR patients who received filgotinib $200 \mathrm{mg}(P<0.001)$ and filgotinib $100 \mathrm{mg}(P \leq 0.013)$ achieved MCID relative to placebo (Fig. 1C). At week $4,60.4 \%$ of bDMARDIR patients who received filgotinib $200 \mathrm{mg}(P<0.001)$ and $54.7 \%$ who received filgotinib $100 \mathrm{mg}(P=0.013)$ achieved MCID compared to $40.3 \%$ of the placebo group.

\section{Differences between treatment groups in proportions of patients achieving HAQ-DI near normal}

The proportion of MTX-naïve patients with HAQDI scores of $\leq 0.5$ at week 4 was $30.8 \%(P<0.001)$ for patients who received filgotinib $200 \mathrm{mg}$ plus MTX, 22.2\% $(P=0.075)$ for filgotinib $100 \mathrm{mg}$ plus MTX, and $28.1 \%$ $(P<0.001)$ for filgotinib $200 \mathrm{mg}$ monotherapy compared with $16.6 \%$ for MTX monotherapy (Fig. 1D). The proportion of patients with HAQ-DI $\leq 0.5$ remained greater for patients receiving any dose of filgotinib relative to MTX alone through week 52 .

Among MTX-IR patients, HAQ-DI $\leq 0.5$ was achieved at week 4 by $18.9 \%(P=0.004)$ of patients who received filgotinib $200 \mathrm{mg}, 15.6 \%(P=0.12)$ receiving filgotinib $100 \mathrm{mg}$, and $12.2 \%$ receiving placebo (Fig. $1 \mathrm{E}$ ). The proportion of bDMARD-IR patients who achieved HAQ-DI $\leq 0.5$ at week 4 was $17.7 \%(P=0.051)$ for filgotinib 200 $\mathrm{mg}, 16.3 \%(P=0.10)$ for filgotinib $100 \mathrm{mg}$, and $10.1 \%$ for placebo. These proportions increased at week 12 for patients who received filgotinib $200 \mathrm{mg}$, filgotinib 100 $\mathrm{mg}$, and placebo (Fig. 1F). This effect was maintained at week 24 for both MTX-IR and bDMARD-IR patients receiving either dose of filgotinib relative to placebo (Fig. 1E, F). The proportion of MTX-IR patients who achieved HAQ-DI $\leq 0.5$ was similar for patients receiving either dose of filgotinib and adalimumab at week 4. A greater proportion of patients who received filgotinib 200 mg achieved HAQ-DI $\leq 0.5$ compared with adalimumab at weeks 12 and 52. Proportions of patients achieving HAQ-DI $\leq 0.5$ were similar for filgotinib $100 \mathrm{mg}$ and adalimumab (Fig. 1E).

\section{Differences between treatment groups in SF-36 changes from baseline}

By week 4, MTX-naïve patients who received filgotinib $200 \mathrm{mg}$ plus MTX $(7.3 \pm 0.4, P<0.001)$, filgotinib $100 \mathrm{mg}$ plus MTX $(5.8 \pm 0.5, P<0.001)$, and filgotinib $200 \mathrm{mg}$ monotherapy $(6.4 \pm 0.5, P=0.001)$ had greater improvements from baseline in SF-36 PCS relative to MTX alone $(4.1 \pm 0.4)$ (Table 1). Patients treated with filgotinib 200 
Table 3 Least-squares mean change from baseline for patientreported outcomes in bDMARD-IR patients; filgotinib dosing regimens compared to placebo

\begin{tabular}{|c|c|c|c|}
\hline PRO measures & $\begin{array}{l}\text { FIL } 200 \mathrm{mg}+ \\
\mathrm{csDMARD} n=147\end{array}$ & $\begin{array}{l}\text { FIL } 100 \mathrm{mg}+ \\
\text { csDMARD } n= \\
153\end{array}$ & $\begin{array}{l}\text { PBO + } \\
\text { csDMARD } n \\
=148\end{array}$ \\
\hline \multicolumn{4}{|l|}{ HAQ-DI } \\
\hline Week 4 & $-0.35(0.05)^{* * *}$ & $-0.28(0.05)^{* *}$ & $-0.13(0.05)$ \\
\hline Week 12 & $-0.50(0.06)^{* * *}$ & $-0.46(0.05)^{* * *}$ & $-0.19(0.06)$ \\
\hline Week 24 & $-0.63(0.06)^{* * *}$ & $-0.49(0.06)^{* *}$ & $-0.27(0.06)$ \\
\hline \multicolumn{4}{|l|}{ SF-36 PCS } \\
\hline Week 4 & $5.9(0.7)^{* * *}$ & $5.4(0.7)^{* *}$ & $3.4(0.7)$ \\
\hline Week 12 & $8.4(0.8)^{* * *}$ & $7.6(0.8)^{* * *}$ & $4.2(0.8)$ \\
\hline Week 24 & $9.6(0.8)^{* * *}$ & $8.9(0.8)^{* *}$ & $5.8(0.9)$ \\
\hline \multicolumn{4}{|l|}{ SF-36 MCS } \\
\hline Week 4 & $2.5(0.9)^{*}$ & $2.0(0.9)$ & $0.3(0.9)$ \\
\hline Week 12 & $4.5(0.9)^{*}$ & $3.5(0.9)$ & $2.5(0.9)$ \\
\hline Week 24 & $4.9(1.0)$ & $3.1(1.0)$ & $3.0(1.1)$ \\
\hline \multicolumn{4}{|l|}{ FACIT-Fatigue } \\
\hline Week 4 & $6.9(1.0)^{* * *}$ & $6.6(1.0)^{* *}$ & $3.3(1.0)$ \\
\hline Week 12 & $10.2(1.0)^{* * *}$ & $8.4(1.0)^{* *}$ & $5.2(1.1)$ \\
\hline Week 24 & $11.5(1.1)^{* * *}$ & $9.0(1.1)$ & $6.9(1.2)$ \\
\hline \multicolumn{4}{|l|}{ PtGA } \\
\hline Week 4 & $-21(2.3)^{* * *}$ & $-20(2.3)^{* * *}$ & $-9(2.4)$ \\
\hline Week 12 & $-31(2.5)^{* * *}$ & $-26(2.5)^{* * *}$ & $-13(2.6)$ \\
\hline Week 24 & $-36(2.6)^{* * *}$ & $-30(2.7)^{* * *}$ & $-18(2.8)$ \\
\hline \multicolumn{4}{|l|}{ WPAI-RA } \\
\hline \multicolumn{4}{|l|}{ Absenteeism } \\
\hline Week 4 & $-5.9(3.5)$ & $-1.3(2.9)$ & $2.4(3.0)$ \\
\hline Week 12 & $-6.9(3.6)^{*}$ & $-3.0(3.0)$ & $3.1(3.2)$ \\
\hline Week 24 & $-5.7(4.4)$ & $-0.5(3.9)$ & $4.1(4.5)$ \\
\hline \multicolumn{4}{|c|}{ Presenteeism } \\
\hline Week 4 & $-22.0(3.9)^{* * *}$ & $-12.5(3.3)$ & $-3.1(3.5)$ \\
\hline Week 12 & $-23.5(4.1)^{* *}$ & $-18.0(3.5)^{*}$ & $-6.8(3.6)$ \\
\hline Week 24 & $-20.9(4.0)$ & $-24.6(3.7)$ & $-15.1(4.0)$ \\
\hline \multicolumn{4}{|c|}{ Work productivity loss } \\
\hline Week 4 & $-22.9(4.1)^{* * *}$ & $-11.3(3.5)$ & $-2.7(3.7)$ \\
\hline Week 12 & $-25.8(4.4)^{* * * *}$ & $-17.7(3.7)^{*}$ & $-5.7(3.9)$ \\
\hline Week 24 & $-21.7(4.6)$ & $-22.8(4.3)$ & $-12.8(4.7)$ \\
\hline \multicolumn{4}{|c|}{ Activity impairment } \\
\hline Week 4 & $-15.9(1.9)^{* * *}$ & $-14.2(1.9)^{* * *}$ & $-4.6(1.9)$ \\
\hline Week 12 & $-24.4(2.1)^{* * *}$ & $-18.8(2.1)^{* *}$ & $-10.5(2.1)$ \\
\hline Week 24 & $-30.1(2.2)^{* * *}$ & $-24.4(2.3)^{* *}$ & $-15.4(2.4)$ \\
\hline
\end{tabular}

Data presented as LS mean (SE). The primary endpoint was assessed at Week 12. Comparison with placebo: ${ }^{* * *} P<0.001,{ }^{* *} P<0.01,{ }^{*} P<0.05$. All $P$ values are exploratory (not adjusted for multiplicity) and assessed using the MMRM, including treatment group, visit, treatment group by visit interaction, stratification factors, baseline value as fixed effects, and patients as random effect. The FINCH 2 study ended at week 24.

CSDMARD, conventional synthetic disease-modifying antirheumatic drug; FACIT, Functional Assessment of Chronic Illness Therapy; FIL, filgotinib; $L S$, least-squares; HAQ-DI, Health Assessment Questionnaire-Disability Index; MCS , Mental Component Score; PBO, placebo; PCS, Physical Component Score; $P R O$ patient-reported outcomes; PtGA , Patient Global Assessment of Disease Activity; SE, standard error; SF-36, Medical Outcomes Study 36-Item Short Form; WPAI-RA, Work Productivity and Activity Impairment Questionnaire-Rheumatoid
Table 3 (continued)

Arthritis

mg plus MTX maintained significant improvements relative to MTX alone through week 52, while such improvements remained significant through week 24 for filgotinib $100 \mathrm{mg}$ plus MTX and through week 12 for filgotinib 200 mg monotherapy. Improvements in SF-36 MCS from baseline were greater among the filgotinib $200 \mathrm{mg}$ plus MTX (4.6 $\pm 0.5, P<0.001)$, filgotinib $100 \mathrm{mg}$ plus MTX (3.4 $\pm 0.6, P=0.032)$, and filgotinib $200 \mathrm{mg}$ monotherapy $(4.3 \pm 0.6, P<0.001)$ groups relative to MTX alone $(1.9 \pm 0.5)$ at week 4 (Table 1$)$. Thereafter, changes from baseline were similar between the filgotinib groups and the MTX alone group.

At week 4 in MTX-IR patients, improvements from baseline in SF-36 PCS and MCS for patients in the filgotinib $200 \mathrm{mg}(6.0 \pm 0.5$ and $3.3 \pm 0.6$, respectively; both $P<0.001)$ and $100 \mathrm{mg}(5.1 \pm 0.5, P<0.001$ and $3.0 \pm$ $0.6, P=0.002$, respectively) groups were greater compared with placebo (3.4 \pm 0.6 and $1.5 \pm 0.6)$; increases were maintained throughout the study (Table 2). MTXIR patients receiving filgotinib $200 \mathrm{mg}$ reported greater improvements relative to adalimumab at weeks 4 and 12 but not at weeks 24 and 52 (Table 2). Among patients receiving filgotinib $100 \mathrm{mg}$, improvements in SF-36 PCS and MCS were similar to those in patients receiving adalimumab throughout the study.

Among bDMARD-IR patients, filgotinib $200 \mathrm{mg}$ provided improvements from baseline compared to placebo for SF-36 PCS at weeks $4(5.9 \pm 0.7$ vs $3.4 \pm 0.7$, $P<0.001), 12(8.4 \pm 0.8$ vs $4.2 \pm 0.8, P<0.001)$, and 24 $(9.6 \pm 0.8$ vs $5.8 \pm 0.9, P<0.001)$ and for SF-36 MCS at weeks $4(2.5 \pm 0.9$ vs $0.3 \pm 0.9, P=0.019)$ and $12(4.5 \pm$ 0.9 vs $2.5 \pm 0.9, P=0.045)$ but not at week 24 . Filgotinib $100 \mathrm{mg}$ improved SF-36 PCS relative to placebo at weeks $4(5.4 \pm 0.7$ vs $3.4 \pm 0.7, P=0.005), 12(7.6 \pm 0.8$ vs $4.2 \pm$ $0.8, P<0.001)$, and $24(8.9 \pm 0.8$ vs $5.8 \pm 0.9, P=0.002)$ (Table 3). Improvements in SF-36 MCS were similar for patients receiving filgotinib $100 \mathrm{mg}$ and placebo throughout the study.

\section{Differences between treatment groups in proportions of patients achieving SF-36 MCID}

At week 4, greater proportions of MTX-naïve patients who received filgotinib $200 \mathrm{mg}$ plus MTX $(70.0 \%, P<$ 0.001), filgotinib $100 \mathrm{mg}$ plus $\operatorname{MTX}(64.7 \%, P=0.004)$, and filgotinib $200 \mathrm{mg}$ monotherapy $(63.9 \%, P=0.006)$ achieved the MCID for SF-36 PCS compared to MTX alone (52.6\%). For filgotinib $200 \mathrm{mg}$ plus MTX compared to MTX alone, significantly greater proportions of patients achieved MCID at weeks 12, 24, and 52; for 

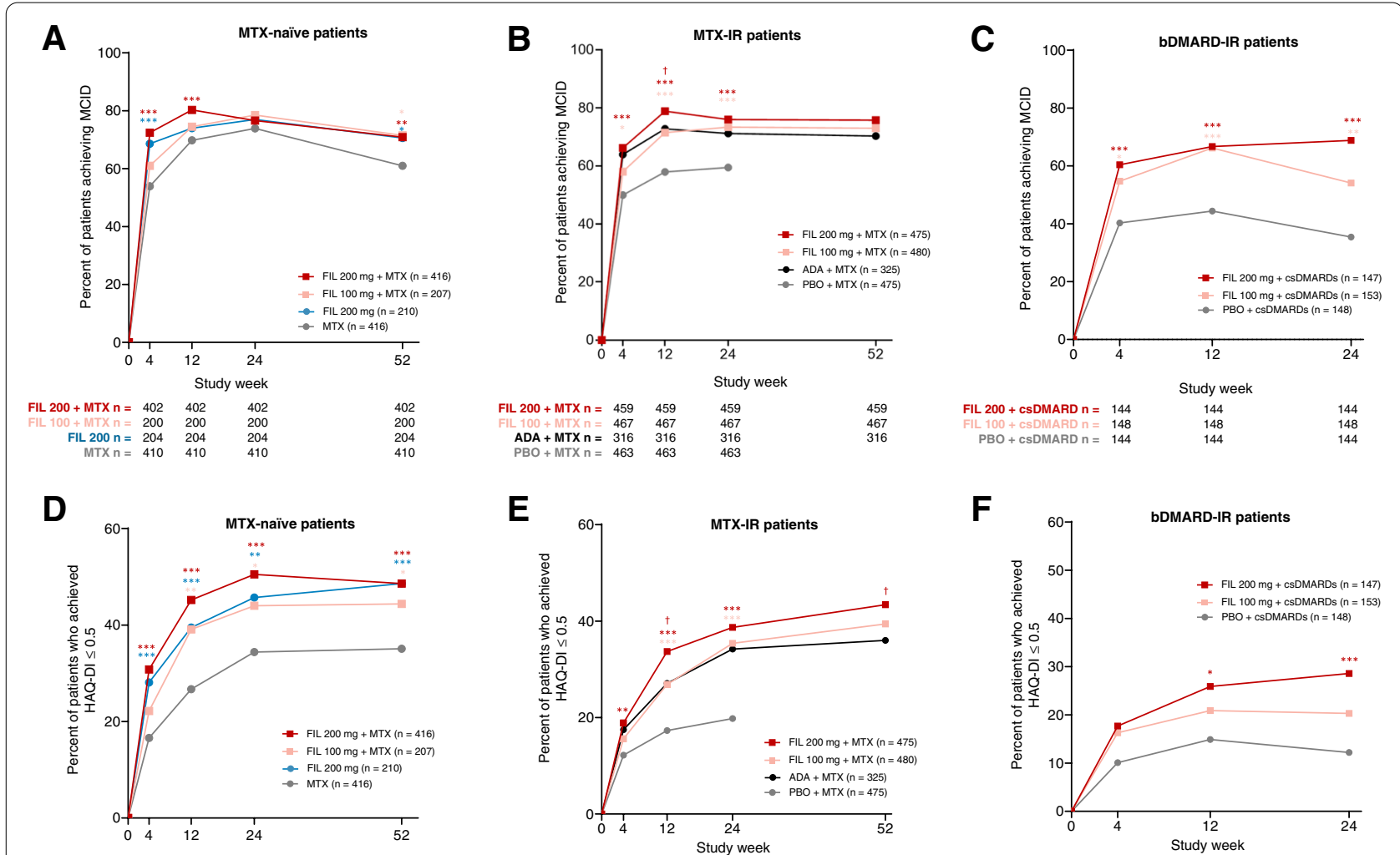

Fig. 1 Proportion of patients achieving MCID for HAQ-DI in A MTX-naïve patients, B MTX-IR patients, and C and bDMARD-IR patients; proportion of patients who achieved HAQ-DI score of $\leq 0.5$ by visit $\mathbf{D}$ MTX-naïve, $\mathbf{E}$ MTX-IR, and $\mathbf{F}$ bDMARD-IR. Comparison with PBO or MTX: ${ }^{* * *} P<$ $0.001,{ }^{* *} P<0.01,{ }^{*} P<0.05$. Comparison with ADA: ${ }^{\dagger} P<0.05$. All $P$ values are exploratory (not adjusted for multiplicity) and were from logistic regression with treatment groups and stratification factors in the model. A nonresponder imputation was used for patients with missing data. MCID was defined as a $\geq 0.22$-point reduction from baseline. In the MTX-IR trial, patients on PBO were rerandomized to FIL 200 or 100 $\mathrm{mg}$ at week 24. The study in bDMARD-IR patients ended at week 24. ADA, adalimumab; bDMARD, biologic DMARD; csDMARD, conventional synthetic DMARD; DMARD, disease-modifying antirheumatic drug; FIL, filgotinib; HAQ-DI, Health Assessment Questionnaire-Disability Index; LS, least-squares; MCID, minimal clinically important difference; MTX, methotrexate; PBO, placebo

filgotinib $100 \mathrm{mg}$ plus MTX, the differences were significant at weeks 12 and 52; and for filgotinib $200 \mathrm{mg}$ as monotherapy, the difference from MTX alone was significant at week 52 . Greater proportions of patients who received filgotinib $200 \mathrm{mg}$ plus MTX $(52.4 \%, P=0.004)$, filgotinib $100 \mathrm{mg}$ plus MTX $(50.2 \%, P=0.060)$, and filgotinib $200 \mathrm{mg}$ monotherapy $(52.4 \%, P=0.016)$ achieved MCID for SF-36 MCS compared to MTX alone (42.3\%) at week 4; at all other timepoints, proportions were similar among filgotinib groups and MTX alone (Fig. 2A, D).

At week $4,65.8 \%(P<0.001)$ of MTX-IR patients who received filgotinib $200 \mathrm{mg}$ and $58.0 \%(P=0.10)$ who received filgotinib $100 \mathrm{mg}$ achieved MCID for SF-36 PCS compared with $52.5 \%$ of the placebo group; differences between the filgotinib groups and placebo were also significant at weeks 12 and 24 . At week $52,74.0 \%$ of patients who received filgotinib $200 \mathrm{mg}$ and $70.6 \%$ who received filgotinib $100 \mathrm{mg}$ reached MCID for SF-36 PCS (Fig. 2B). In MTX-IR patients, $53.7 \%(P=0.002)$ of the filgotinib 200 and $52.6 \%(P=0.007)$ of the filgotinib $100 \mathrm{mg}$ groups achieved MCID for SF-36 MCS compared to $44.1 \%$ of the placebo group at week 4 (Fig. 2E); differences remained significant between filgotinib groups and placebo at weeks 12 and 24. The proportions of MTX-IR patients in the filgotinib groups who achieved MCID for SF-36 PCS and MCS were similar to those of the adalimumab group throughout the study.

Among bDMARD-IR patients, the proportion of patients achieving MCID was higher for patients in the filgotinib $200 \mathrm{mg}$ group relative to placebo at weeks $4(62.3 \%$ vs $48.0 \%, P=0.010), 12$ (76.0\% vs $47.3 \%, P<0.001)$, and 24 (67.8\% vs $40.5 \%, P<0.001)$ for SF-36 PCS and was similar to placebo until week $24(52.1 \%$ vs $32.4 \%, P<0.001)$ for SF-36 MCS (Fig. 2C, F). Among patients who received filgotinib $100 \mathrm{mg}$ compared with placebo, the proportion 


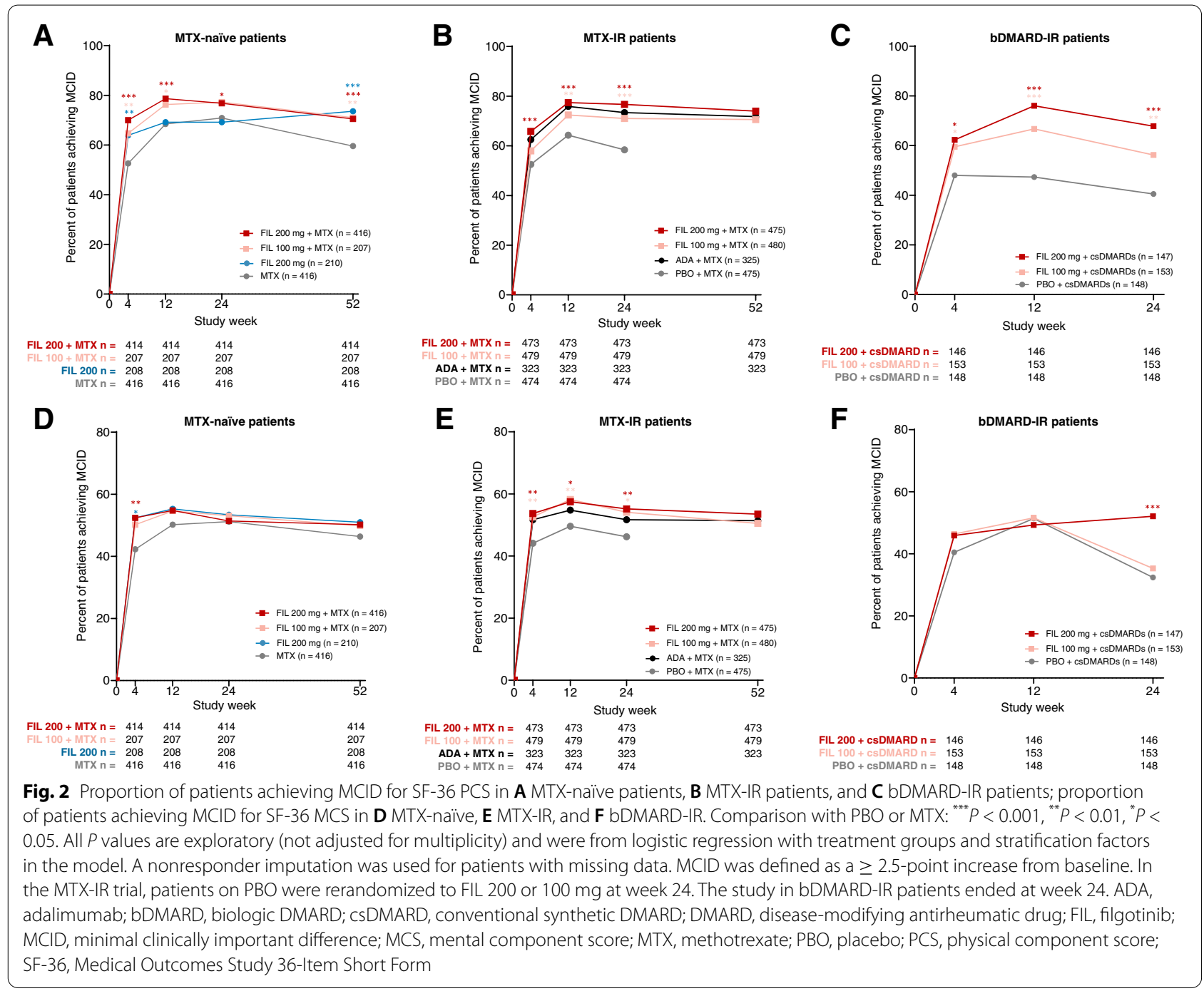

who achieved MCID for SF-36 PCS was higher at weeks 4 (59.5\% vs $48.0 \%, P=0.049), 12(66.7 \%$ vs $47.3 \%, P<$ $0.001)$, and 24 (56.2\% vs $40.5 \%, P=0.007)$ and was similar to placebo throughout the study for SF-36 MCS.

In all 3 studies, improvements from baseline in individual SF-36 domain scores were evident as early as week 4 for MTX-naïve, MTX-IR, and bDMARD-IR patients treated with either dose of filgotinib and persisted throughout treatment until week 52 (Fig. 3).

\section{Differences between treatment groups in FACIT-Fatigue changes from baseline}

At week 4, MTX-naïve patients who received filgotinib 200 or $100 \mathrm{mg}$ plus MTX and filgotinib $200 \mathrm{mg}$ monotherapy reported significantly greater improvements in FACIT-Fatigue compared to MTX alone. Improvements were maintained through week 52 (Table 1). By week 4, MTX-IR patients who received filgotinib 200 or $100 \mathrm{mg}$ demonstrated significant improvements from baseline in FACIT-Fatigue scores relative to placebo as did bDMARD-IR patients who received filgotinib 200 or $100 \mathrm{mg}$. Improvements were maintained through week 52 and week 24 for MTX-IR and bDMARDIR patients, respectively (Tables 2 and 3). FACITFatigue scores were numerically greater in MTX-IR patients receiving any dose of filgotinib compared with adalimumab.

\section{Differences between treatment groups in proportions of patients achieving FACIT-Fatigue MCID}

Among MTX-naïve patients, the MCID for FACITFatigue was achieved by $60.3 \%$ of the filgotinib $200 \mathrm{mg}$ plus MTX $(P<0.001), 57.6 \%$ of the filgotinib $100 \mathrm{mg}$ plus MTX $(P=0.025)$, and $57.6 \%$ of the filgotinib 200 


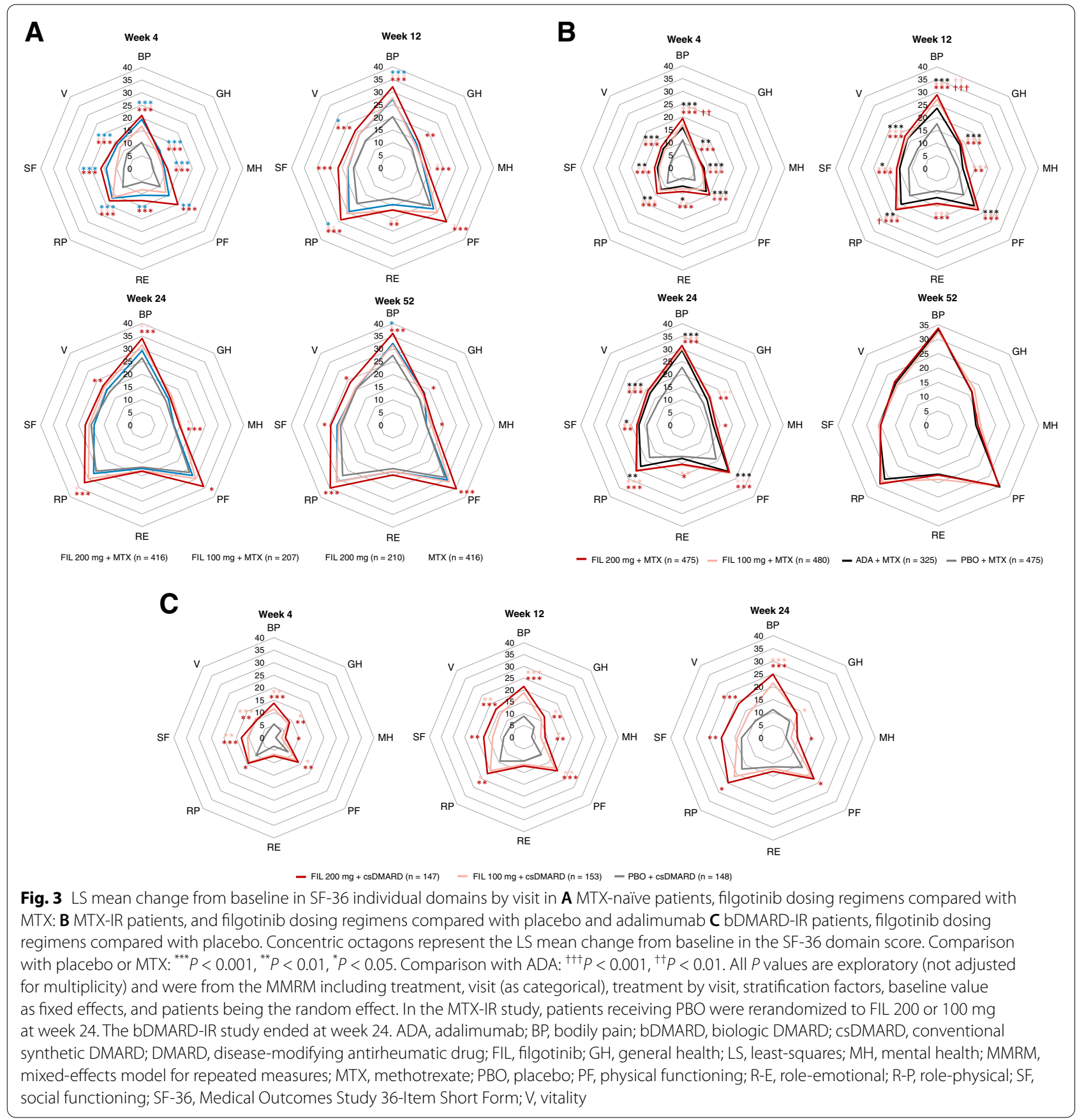

mg monotherapy $(P=0.033)$ groups relative to $48.4 \%$ of the MTX group (Fig. 4A) at week 4; proportions achieving MCID were similar across groups at weeks 12, 24, and 52. Among MTX-IR patients, at weeks 4 and $24,57.8 \%(P=0.002)$ and $64.6 \%(P<0.001)$ of patients who received filgotinib $200 \mathrm{mg}$ and $58.1 \%(P=0.002)$ and $63.7 \%(P<0.001)$ of patients who received filgotinib $100 \mathrm{mg}$ achieved MCID relative to $48 \%$ and $52.7 \%$ of the placebo group. The percentage of patients treated with filgotinib who achieved MCID remained consistent through week 52 (Fig. 4B). Results were similar between patients who received either dose of filgotinib and adalimumab throughout the study (Fig. 4B). At week 4 , a greater proportion of bDMARD-IR patients who received filgotinib $200 \mathrm{mg}(56.3 \%, P=0.013)$ and filgotinib $100 \mathrm{mg}(59.7 \%, P=0.003)$ achieved MCID relative to placebo (41.7\%), and at week 24 , a greater proportion of patients receiving filgotinib $200 \mathrm{mg}(75.4 \%, P=$ 

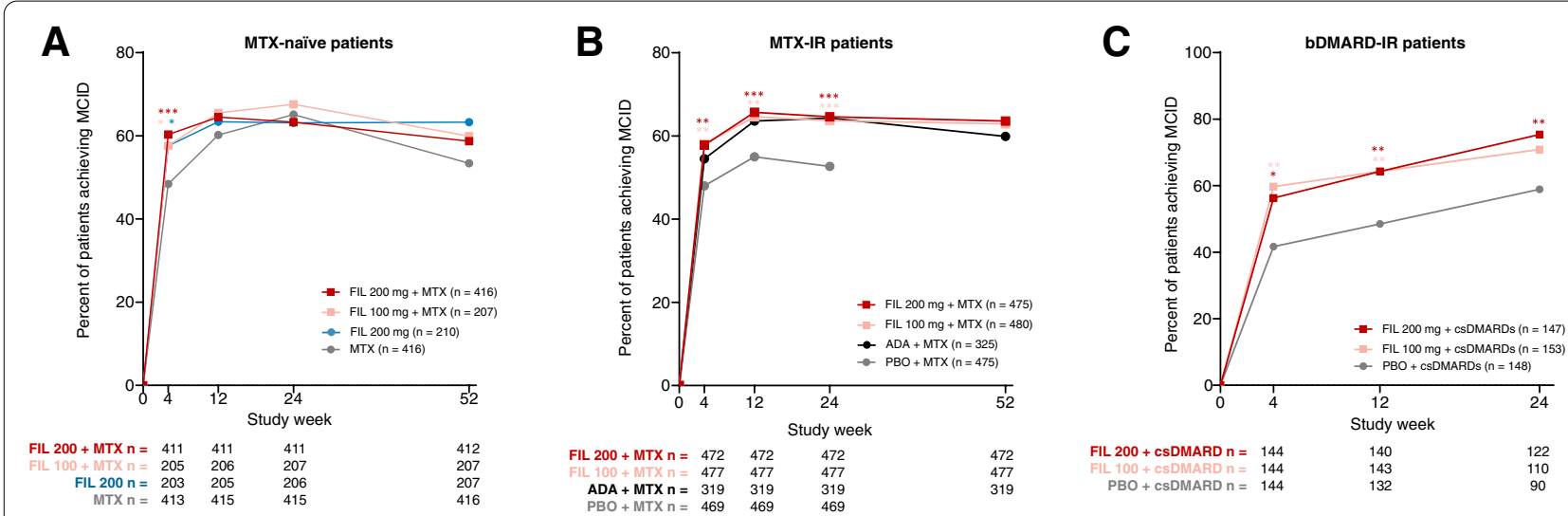

Fig. 4 Proportion of patients achieving MCID for FACIT-Fatigue in A MTX-naïve patients, B MTX-IR patients, and C bDMARD-IR patients. Comparison with placebo or methotrexate: ${ }^{* * *} P<0.001,{ }^{* *} P<0.01,{ }^{*} P<0.05$. Comparison with adalimumab: ${ }^{\dagger} P<0.05$. All $P$ values are exploratory (not adjusted for multiplicity) and were from logistic regression with treatment groups and stratification factors in the model. A nonresponder imputation was used for patients with missing data. MCID was defined as a $\geq 4$-point increase from baseline. In the MTX-IR study, patients on PBO were rerandomized to FIL 200 or 100 mg at week 24. The bDMARD-IR study ended at week 24. ADA, adalimumab; bDMARD, biologic DMARD; cSDMARD, conventional synthetic DMARD; DMARD, disease-modifying antirheumatic drug; FACIT, Functional Assessment of Chronic IIIness Therapy; FIL, filgotinib; MCID, minimal clinically important difference; MTX, methotrexate; PBO, placebo

0.008 ), but not filgotinib $100 \mathrm{mg}$, achieved MCID vs placebo (58.9\%) (Fig. 4C).

\section{Differences between treatment groups in WPAI-RA changes from baseline}

At baseline, the percentage of employed MTX-naïve patients in the filgotinib $200 \mathrm{mg}$ plus MTX, filgotinib 100 mg plus MTX, filgotinib $200 \mathrm{mg}$ monotherapy, and MTX groups were $42.3 \%, 39.6 \%, 44.7 \%$, and $41.2 \%$, respectively. At week 4, least-squares mean change from baseline was significantly greater for filgotinib 200 and $100 \mathrm{mg}$ plus MTX and for filgotinib monotherapy relative to MTX monotherapy for presenteeism, work productivity loss, and activity impairment. Changes in absenteeism were similar between patients receiving any dose of filgotinib and MTX alone. Improvements were maintained through week 52 (Table 1).

At baseline, the percentage of employed MTX-IR patients in the filgotinib $200 \mathrm{mg}$, filgotinib $100 \mathrm{mg}$, adalimumab, and placebo groups were $42.8 \%, 41.5 \%, 41.1 \%$, and $35.6 \%$, respectively. Patients in the filgotinib 200 mg group reported significantly greater improvements relative to placebo at weeks 4,12 , and 24 in presenteeism, work productivity loss, and activity impairment. Improvements in absenteeism were similar between patients receiving filgotinib $200 \mathrm{mg}$ and placebo at weeks 4 and 12 but greater at week 24 for patients receiving filgotinib $200 \mathrm{mg}$ (Table 2).

Among MTX-IR patients receiving filgotinib 100 $\mathrm{mg}$, improvements relative to placebo were similar at week 4 but significantly greater at weeks 12 and 24 for presenteeism and work productivity loss. Improvements were significantly greater for patients receiving filgotinib $100 \mathrm{mg}$ relative to placebo at weeks 4,12 , and 24 for activity impairment. For absenteeism, improvements were similar between patients receiving filgotinib $100 \mathrm{mg}$ and placebo at weeks 4 and 12 but greater at week 24 for patients receiving filgotinib $100 \mathrm{mg}$ (Table 2). Improvements in presenteeism, work productivity loss, and activity impairment were maintained through 52 weeks (Table 2). Results were similar compared with adalimumab.

At baseline, the percentages of employed bDMARD-IR patients receiving filgotinib $200 \mathrm{mg}(24.0 \%)$, filgotinib 100 $\mathrm{mg}(35.5 \%)$, and placebo (36.1\%) were lower compared to the MTX-IR and MTX-naïve study populations. Among bDMARD-IR patients who received filgotinib $200 \mathrm{mg}$, absenteeism was similar to patients receiving placebo at weeks 4 and 24 but significantly improved relative to placebo at week 12 . Improvements for patients receiving filgotinib $200 \mathrm{mg}$ relative to placebo were significantly greater at weeks 4 and 12 for presenteeism and work productivity but not at week 24 . Activity impairment was significantly improved relative to placebo at weeks 4,12 , and 24 (Table 3). For bDMARD-IR patients who received filgotinib $100 \mathrm{mg}$, presenteeism and work productivity loss were significantly improved relative to placebo at week 12, but improvements were similar at weeks 4 and 24. Activity impairment was significantly improved at weeks 4,12 , and 24 (Table 3 ). 


\section{Differences between treatment groups in PtGA changes from baseline}

In all 3 studies, MTX-naïve, MTX-IR, and bDMARD-IR patients experienced rapid and sustained improvements in patient-assessed disease activity (Tables 1, 2, and 3). MTX-naïve patients who received filgotinib reported significantly greater improvements in PtGA at week 4 relative to MTX monotherapy, and improvements continued and were maintained through week 52 (Table 1). Similarly, MTX-IR patients who received filgotinib reported significantly greater improvements relative to placebo at weeks 4,12 , and 24 (Table 2). With the exception of a significantly greater reduction from baseline among patients receiving filgotinib $200 \mathrm{mg}$ relative to adalimumab at week 12, improvements in PtGA were similar for either dose of filgotinib and adalimumab throughout the study. bDMARD-IR patients who received filgotinib $200 \mathrm{mg}$ and filgotinib $100 \mathrm{mg}$ reported significant improvements relative to placebo at all timepoints (Table 3 ).

\section{Discussion}

Once-daily filgotinib 200 or $100 \mathrm{mg}$ in combination with MTX or with csDMARDs provided rapid and sustained improvements in functional status, HRQL, fatigue, presenteeism, work productivity, and disease activity as reported by patients with RA who were MTX-naïve or who had inadequate response to MTX or bDMARDs. Filgotinib in combination with MTX provided improvements at levels similar to or better than adalimumab plus MTX for patients with an inadequate response to MTX for up to 52 weeks. Improvements in functional status, fatigue, and physical HRQL were beyond the minimally important differences for many patients, and a higher proportion experienced minimally important differences with either dosage of filgotinib compared with placebo. In general, in these exploratory analyses, therapy with filgotinib $200 \mathrm{mg}$ plus MTX or csDMARDs appeared to provide a greater benefit in each PRO measured for MTX-naive, MTX-IR, and bDMARDIR patients. The results of this analysis were similar to those observed in other Phase 3 trials of the JAK inhibitors tofacitinib, baricitinib, and upadacitinib [16-18, 21-26].

A key strength of this analysis is the inclusion of 3 separate clinical trials that evaluated filgotinib in different patient populations. This allowed us to better evaluate the effect of filgotinib in an MTX-naïve patient population relative to patients with inadequate response to csDMARDs (MTX) or bDMARDs. Limitations of the study include that all analyses and $P$ values, with the exception of HAQ-DI mean change from baseline at the primary timepoints, were exploratory. Accordingly, although improvements relative to the placebo or active controls were reported, the statistical significance of the changes should be interpreted with caution. Second, although 3 patient populations were included, statistical comparisons of filgotinib treatment were not performed between studies, and any conclusions regarding the relative effectiveness of filgotinib in one patient population vs another are conjectural. Third, the bDMARD-IR trial was limited in duration to 24 weeks and enrolled a smaller number of patients, which precludes conclusions regarding duration of benefit among bDMARD-IR patients.

\section{Conclusions}

Filgotinib improved patient functional status, HRQL, fatigue, work impairment, and assessments of disease activity in multiple patient populations. These PRO results suggest filgotinib used as monotherapy or in combination can be an effective treatment option for patients with insufficient response to MTX or bDMARDs, as well as for patients who are MTX-naïve. Additional analyses of the different RA patient populations with longterm treatment will better define the role of filgotinib in improving HRQL in MTX-naïve and in treatmentrefractory populations. An extension trial (FINCH 4, NCT03025308) to evaluate the long-term outcomes of patients who completed these 3 studies is ongoing.

\section{Abbreviations}

ACR: American College of Rheumatology; bDMARD-IR: Biologic disease-modifying antirheumatic drug; csDMARD: Conventional synthetic disease-modifying antirheumatic drug; FACIT-Fatigue: Functional Assessment of Chronic IIIness Therapy-Fatigue; HAQ-DI: Health Assessment Questionnaire-Disability Index; HRQL: Health-related quality of life; IR: Inadequate response; JAK1: Janus kinase 1; MCID: Minimal clinically important difference; MCS: Mental Component Summary (SF-36); MMRM: Mixed-effects model for repeated measures; MTX-IR: Methotrexate; PCS: Physical Component Summary (SF-36); PRO: Patient-reported outcome; PtGA: Patient Global Assessment of Disease Activity; RA: Rheumatoid arthritis; SF-36: Medical Outcomes Study 36-Item Short Form; SE: Standard error; WPAI-RA: Work Productivity and Activity Impairment Questionnaire-Rheumatoid Arthritis.

\section{Supplementary Information}

The online version contains supplementary material available at https://doi. org/10.1186/s13075-021-02677-7.

Additional file 1: Supplementary Table 1. Patient demographics and baseline characteristics, MTX-naïve trial.

Additional file 2: Supplementary Table 2. Patient demographics and baseline characteristics, MTX-IR trial.

Additional file 3: Supplementary Table 3. Patient demographics and baseline characteristics, bDMARD-IR trial.

\section{Acknowledgements}

Medical writing support was provided by Shavonn Harper, PhD, and Gregory Bezkorovainy, MA, of AlphaScientia, LLC, San Francisco, CA; and funded by Gilead Sciences, Inc., Foster City, CA.

\section{Authors' contributions}

LY performed the statistical analyses. All authors contributed to the interpretation of the results, critically reviewed the manuscript during writing, and 
approved of the final draft for submission. The author(s) read and approved the final manuscript.

\section{Funding}

This study was funded by Gilead Sciences, Inc., Foster City, CA.

\section{Availability of data and materials}

Data are available upon reasonable request. Anonymized individual patient data will be shared upon request for research purposes dependent upon the nature of the request, the merit of the proposed research, the availability of the data, and the intended use. The full data sharing policy for Gilead Sciences can be found at https://www.gilead.com/about/ethics-and-code-of-conduct/policies.

\section{Declarations}

\section{Ethics approval and consent to participate}

All trials were conducted in accordance with the Declaration of Helsinki and International Council for Harmonisation Good Clinical Practice guidelines. FINCH 1 was approved by the Advarra Central Institutional Review Board (Reference \# 00000971). FINCH 2 was approved by the Administrative Panel on Human Subjects in Medical Research (Reference \# 4593). FINCH 3 was approved by Ethics Committee Research UZ/KU Leuven (Reference \# S59627). The study protocols were approved by the international review board or ethics committee at each study site, and all patients provided written informed consent.

\section{Consent for publication}

Not applicable.

\section{Competing interests}

COB reports grant/research support from BMS; is a consultant for AbbVie; BMS; Eli Lilly \& Co.; Gilead Sciences, Inc.; Janssen; Pfizer; Regeneron; and Sanofi. DW has financial ties with Eli Lilly \& Co.; Gilead Sciences, Inc.; Pfizer; and Roche. PN reports financial relationships with AbbVie, BMS, Boehringer Ingelheim, Celgene, Eli Lilly \& Co., Gilead, Janssen, MSD, Novartis, Pfizer, Roche, and Sanofi. SJL, $L Y$, and $\mathrm{HH}$ are employees and shareholders of Gilead Sciences, Inc. JMK is an employee of Galapagos NV. BC receives grant/research support from Novartis, Pfizer, and Roche-Chugai; is a consultant for AbbVie; Celltrion; Eli Lilly \& Co.; Galapagos; Gilead Sciences, Inc.; Janssen; Roche-Chugai; and is on the speakers bureau for BMS; Eli Lilly \& Co.; Gilead Sciences, Inc.; MSD; Pfizer; Roche-Chugai.

\section{Author details}

'Division of Rheumatology, Johns Hopkins University, 5200 Eastern Avenue, Mason F. Lord Bldg, Center Tower, Room 434A, Baltimore, MD 21224, USA. ${ }^{2}$ Northumbria Healthcare Trust, North Shields, UK. ${ }^{3}$ Griffith University, Brisbane, QLD, Australia. ${ }^{4}$ Gilead Sciences, Inc., Foster City, CA, USA. ${ }^{5}$ Galapagos BV, Leiden, Netherlands. ${ }^{6}$ University of Montpellier, Montpellier, France.

Received: 25 May 2021 Accepted: 9 November 2021

Published online: 03 January 2022

\section{References}

1. Genovese MC, Kalunian K, Gottenberg JE, et al. Effect of filgotinib vs placebo on clinical response in patients with moderate to severe rheumatoid arthritis refractory to disease-modifying antirheumatic drug therapy: the FINCH 2 Randomized Clinical Trial. JAMA. 2019;322(4):315-25.

2. Pincus T, Callahan LF, Sale WG, Brooks AL, Payne LE, Vaughn WK. Severe functional declines, work disability, and increased mortality in seventyfive rheumatoid arthritis patients studied over nine years. Arthritis Rheum. 1984;27(8):864-72.

3. Felson DT, Anderson JJ, Boers M, et al. The American College of Rheumatology preliminary core set of disease activity measures for rheumatoid arthritis clinical trials. The Committee on Outcome Measures in Rheumatoid Arthritis Clinical Trials. Arthritis Rheum. 1993;36(6):729-40.

4. Strand V, Mease P, Burmester GR, et al. Rapid and sustained improvements in health-related quality of life, fatigue, and other patient-reported outcomes in rheumatoid arthritis patients treated with certolizumab pegol plus methotrexate over 1 year: results from the RAPID 1 randomized controlled trial. Arthritis Res Ther. 2009;11(6):R170.

5. Van Rompaey L, Galien R, van der Aar EM, et al. Preclinical characterization of GLPG0634, a selective inhibitor of JAK1, for the treatment of inflammatory diseases. J Immunol. 2013;191(7):3568-77.

6. Combe B, Kivitz A, Tanaka Y, et al. Filgotinib versus placebo or adalimumab in patients with rheumatoid arthritis and inadequate response to methotrexate: a phase III randomised clinical trial. Ann Rheum Dis. 2021;80:848-58.

7. Westhovens R, Rigby WFC, van der Heijde D, et al. Filgotinib in combination with methotrexate or as monotherapy versus methotrexate monotherapy in patients with active rheumatoid arthritis and limited or no prior exposure to methotrexate: the phase 3 , randomised controlled FINCH 3 trial. Ann Rheum Dis. 2021;80:727-38.

8. Strand V, Gossec L, Proudfoot CWJ, et al. Patient-reported outcomes from a randomized phase III trial of sarilumab monotherapy versus adalimumab monotherapy in patients with rheumatoid arthritis. Arthritis Res Ther. 2018;20(1):129.

9. Cole JC, Motivala SJ, Khanna D, Lee JY, Paulus HE, Irwin MR. Validation of single-factor structure and scoring protocol for the Health Assessment Questionnaire-Disability Index. Arthritis Rheum. 2005;53(4):536-42.

10. Tugwell P, Wells $G$, Strand $V$, et al. Clinical improvement as reflected in measures of function and health-related quality of life following treatment with leflunomide compared with methotrexate in patients with rheumatoid arthritis: sensitivity and relative efficiency to detect a treatment effect in a twelve-month, placebo-controlled trial. Leflunomide Rheumatoid Arthritis Investigators Group. Arthritis Rheum. 2000;43(3):506-14.

11. Cella D, Yount S, Sorensen M, Chartash E, Sengupta N, Grober J. Validation of the Functional Assessment of Chronic Illness Therapy Fatigue Scale relative to other instrumentation in patients with rheumatoid arthritis. J Rheumatol. 2005;32(5):811-9.

12. Reilly MC, Zbrozek AS, Dukes EM. The validity and reproducibility of a work productivity and activity impairment instrument. Pharmacoeconomics. 1993;4(5):353-65.

13. Reilly Associates WPAl general information. http://www.reillyassociates. net/WPAI_General.html. Accessed 20 Jan 2021.

14. Zhang W, Bansback N, Boonen A, Young A, Singh A, Anis AH. Validity of the work productivity and activity impairment questionnaire--general health version in patients with rheumatoid arthritis. Arthritis Res Ther. 2010;12(5):R177.

15. Kim D, Kaneko Y, Takeuchi T. Importance of obtaining remission for work productivity and activity of patients with rheumatoid arthritis. J Rheumatol. 2017:44(8):1112-7.

16. Emery P, Blanco R, Maldonado Cocco J, et al. Patient-reported outcomes from a phase III study of baricitinib in patients with conventional synthetic DMARD-refractory rheumatoid arthritis. RMD Open. 2017;3(1):e000410.

17. Smolen JS, Kremer JM, Gaich CL, et al. Patient-reported outcomes from a randomised phase III study of baricitinib in patients with rheumatoid arthritis and an inadequate response to biological agents (RA-BEACON). Ann Rheum Dis. 2017;76(4):694-700.

18. Keystone EC, Taylor PC, Tanaka Y, et al. Patient-reported outcomes from a phase 3 study of baricitinib versus placebo or adalimumab in rheumatoid arthritis: secondary analyses from the RA-BEAM study. Ann Rheum Dis. 2017:76(11):1853-61.

19. Wells GA, Tugwell P, Kraag GR, Baker PR, Groh J, Redelmeier DA. Minimum important difference between patients with rheumatoid arthritis: the patient's perspective. J Rheumatol. 1993;20(3):557-60.

20. Wells G, Li T, Maxwell L, Maclean R, Tugwell P. Responsiveness of patient reported outcomes including fatigue, sleep quality, activity limitation, and quality of life following treatment with abatacept for rheumatoid arthritis. Ann Rheum Dis. 2008;67(2):260-5.

21. Strand $V$, van der Heijde D, Tanaka Y, et al. Tofacitinib in combination with methotrexate in patients with rheumatoid arthritis: patient-reported outcomes from the 24-month Phase 3 ORAL Scan study. Clin Exp Rheumatol. 2020;38(5):848-57.

22. Strand V, Mysler E, Moots RJ, et al. Patient-reported outcomes for tofacitinib with and without methotrexate, or adalimumab with methotrexate, in rheumatoid arthritis: a phase IIIB/IV trial. RMD Open. 2019;5(2):e001040,

23. Strand V, Schiff M, Tundia N, et al. Effects of upadacitinib on patient-reported outcomes: results from SELECT-BEYOND, a phase 3 randomized trial in patients with rheumatoid arthritis and inadequate responses to biologic disease-modifying antirheumatic drugs. Arthritis Res Ther. 2019;21(1):263. 
24. Strand V, Pope J, Tundia N, et al. Upadacitinib improves patient-reported outcomes in patients with rheumatoid arthritis and inadequate response to conventional synthetic disease-modifying antirheumatic drugs: results from SELECT-NEXT. Arthritis Res Ther. 2019;21(1):272.

25. Strand $\mathrm{V}$, Tundia N, Wells A, et al. Upadacitinib monotherapy improves patient-reported outcomes in rheumatoid arthritis: results from SELECTEARLY and SELECT-MONOTHERAPY. Rheumatology (Oxford). 2020. https://doi.org/10.1093/rheumatology/keaa770.

26. Strand V, Tundia N, Bergman M, et al. Upadacitinib improves patientreported outcomes vs placebo or adalimumab in patients with rheumatoid arthritis: results from SELECT-COMPARE. Rheumatology (Oxford). 2021. https://doi.org/10.1093/rheumatology/keab158.

\section{Publisher's Note}

Springer Nature remains neutral with regard to jurisdictional claims in published maps and institutional affiliations.

- fast, convenient online submission

- thorough peer review by experienced researchers in your field

- rapid publication on acceptance

- support for research data, including large and complex data types

- gold Open Access which fosters wider collaboration and increased citations

- maximum visibility for your research: over $100 \mathrm{M}$ website views per year

At BMC, research is always in progress.

Learn more biomedcentral.com/submissions 